\title{
The Feasibility Analysis of Communication Service Model for Power Wireless Private Network with 4G Technology
}

\author{
Hao Zhang ${ }^{1}$, Jiming $\mathrm{Yao}^{1}$, Hao $\mathrm{He}^{2}$ and $\mathrm{Xin} \mathrm{Li}^{2}$ \\ ${ }^{1}$ State Grid Smart Grid Research Institute, No.8 NARI Road, Nanjing, 210003 \\ ${ }^{2}$ Jiangsu Posts \& Telecommunications Planning and Designing Institute CO, LTD, No.58 Nanxijiangdong Street, Nanjing, 210019
}

\begin{abstract}
Smart grid is a high-speed, two-way, real-time, and integrated communication system, which can real-time monitor and control grid operation, prevent accident and clear fault timely. A key component of the Smart Grid is a communications infrastructure for data acquisition, monitoring, control and protection. As a popular wireless broadband communication technology, the Long Term Evolution (LTE) radio access technology defined by the 3rd Generation Partnership Project (3GPP) provides a promising solution for communication networks used in smart grid. Based on the characteristics of new generation broadband wireless communications technologies and wireless communications demands in smart grid, the smart grid wireless communications network is proposed. Since the TDDLTE technology more suitable for relatively small bandwidth and asymmetric traffic. Therefore, we conclude that TD-LTE is a promising candidate to build a smart grid d wireless communications network.
\end{abstract}

Keywords-smart grid; LTE; TDD; requirements

\section{INTRODUCTION}

As a fundamental part of the national energy industry chain, gird plays an important role in the national economy. Every walk of life requests higher demands for an efficiency, reliability and safety power gird. A new concept of next generation electric power system that will feature advanced configurability, reactiveness, and self-management. The smart grid is a modern electric power grid infrastructure for improved efficiency, reliability and safety, with smooth integration of renewable and alternative energy sources, through automated control and modern communications technologies [1], [2].Through shared information on requirements and capabilities among power providers, distributors and consumers, electricity usages can be dynamically and intelligently adjusted, so that the grid network is more efficient and reliable [3], [4].

On the other hand, all of smart grid links require high-speed, bi-directional, real-time, integrated communication networks to support reliable data exchange. LTE has attached sufficient attention since its first commercial release (release 8) was finalized in March 2009 by 3GPP [5]. As a transition between 3G and 4G technology, LTE can achieve uplink peak rate $50 \mathrm{Mb} / \mathrm{s}$ and downlink $100 \mathrm{Mb} / \mathrm{s}$; LTE-Advanced is generally considered as industry standard for 4G, with the uplink peak rate up to $500 \mathrm{Mb} / \mathrm{s}$ and downlink $1 \mathrm{~Gb} / \mathrm{s}$. 4G communication networks has the following advantages: economies of scale associated with a global standard, wide area wireless support across a power transmission and/or distribution network, efficient use of radio resources due to packet switching, support for applications with different Quality-of-Service (QoS) requirements, built-in security function and a flat IP based architecture[5]. 4G has attracted considerable interest for deployment as the wide area communications network in a Smart Grid environment owing to abovementioned characteristics $[6,7]$.

In this paper, we presents four key links of smart grid communications system based on 4G technology. The feasibility of applying $4 \mathrm{G}$ in power generation, transmission, substation, distribution and utilization are analyzed.

\section{REQUIREMENTS AND TRAFFIC IN SMART GRID}

\section{A. Power Transmission Lines}

Transmission lines easily threaten by the climate, geology, human factors, the annual pollution flashover, lightning, ice, dancing and other disasters which caused a great loss to power grid. Transmission lines running state decided power system security and efficiency directly. Hence transmission line monitoring and disaster monitoring shows enormous significance to the realization of the intelligent transmission. However, traditional monitoring method cannot satisfy the new requirements. The establishment of a reliable performance, stability, security, feature-rich transmission line state monitoring and control system is the necessary condition to improve the efficiency of power transmission, is also the premise and foundation to realize smart transmission. According to the current development situation of transmission line, intelligent power transmission management has the following requirements:

(1) Transmission line condition monitoring. Through the image, video and other information collection technology to realize all-round monitoring meteorological environment, ice, temperature, sag and monsoon for transmission line.

(2) Improve the transmission capacity, reduce the transmission loss and optimize the transmission structure through online monitoring technology, realize intelligent fault isolation, fault self-healing and higher equipment utilization level eventually. 


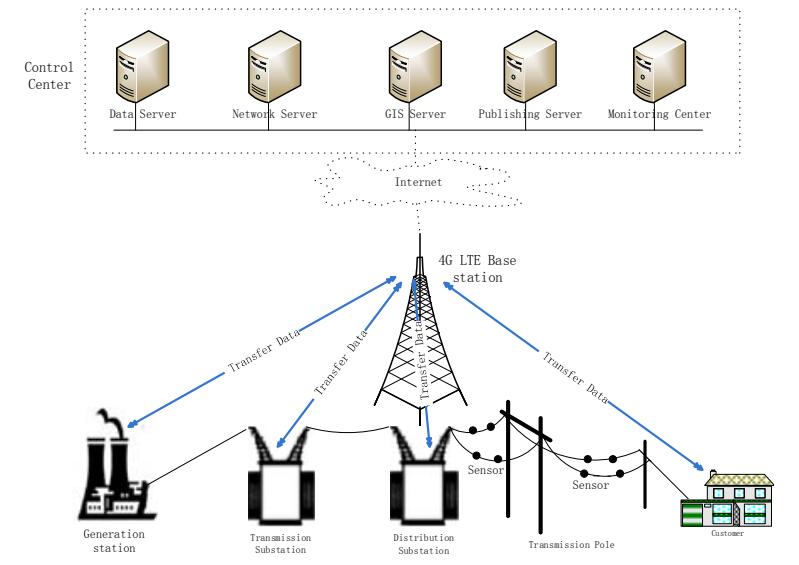

FIGURE I . 4G ENABLED SMART GRID COMMUNICATION SYSTEM

(3) For long distance power transmission, increase the capacity and voltage level to would improve the transmission efficiency. On the other hand, increase the capacity and voltage leads some new challenges and requirements. Such as tide control, system diagnosis and the voltage more unstable, to solve these problems depends on wireless monitoring, analysis and control technology.

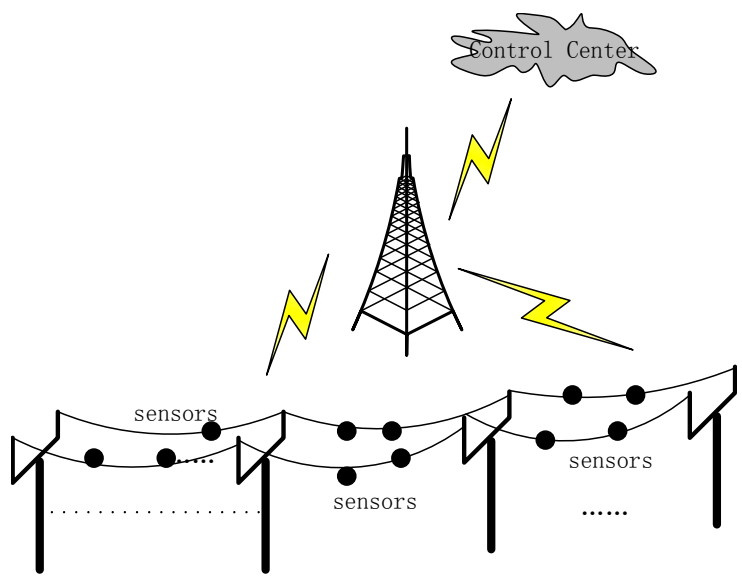

FIGURE II . TRANSMISSION IN SMART GRID

The communication service of transmission line such as relay protection data, security and stability control always occupies an important position in electric power communication network. With the flexible alternative current transmission technology be widely used in power transmission line, the transmission capacity and voltage, power flow control flexibility would significant improved.

To realize the real-time online monitoring of transmission lines service, some advanced measurement, sensing, control technology should be employed for data acquisition. More importantly, communication technology should be used to guarantee the data transmission between the control center and power lines.

Filth insulator. In order to reduce or eliminate the defiled flashover, if's necessary to real-time monitoring the line insulator string of leakage current, environment temperature and humidity, wind speed, wind and rain in real-time monitoring.

Dancing of power transmission line. Dancing can cause line tripping, burns wire, wire broken. Hence the environmental parameters of the transmission line and the parameters of the conductor (tension, lead single, span, etc.) should be monitored in real-time.

Conductor temperature and the online dynamic capacity. Monitoring conductor status (wire temperature, tension and sag, etc.) and meteorological conditions (temperature, sunshine, wind speed, etc.), according to the mathematical model to calculate the maximum available capacity of the wire, improve the transmission capacity of transmission lines.

Lightning arrester. Transmission lines has a high probability struck by lightning, through real-time collecting the leakage current, voltage, lightning frequency, the lightning tripout rate of transmission lines can be greatly reduced.

Digital substation is the foundation of smart grid. Intelligent primary equipment and informationalized secondary equipment is the essence of digital substation. Primary equipment is the basic unit of the power grid, its intelligent degree is related to the overall level of the smart grid.

\section{B. Substation}

Traditional substation collect the analog data of primary equipment to monitor and control primary equipment, use cable for data transmission. Therefore, primary equipment have a weak reliability, such as insulation fault, refusing action or error action. Moreover, for the existing automation system, no interoperability between the devices, poor system scalability and reliability etc., all these problems should be solved in the further. On the other hand, there are a lot of electrical equipment in substation, all sorts of reasons would cause electrical equipment overheating. Especially the overheating caused by equipment abnormal, if can't be handled in time, it would cause catastrophe.

In view of the above analysis, the power substation has the following requirements:

(1) Intelligent primary equipment. Employing wireless sensor network technology, information collection technology of network layer, information processing and intelligent evaluation, information interaction technology of application layer in risk assessment of equipment accident. Optimize the decision in grid operation and equipment maintenance. Improve the observability, controllability and selfrecoverability in intellectualized operation of power grid components.

(2) Automatic operation and management. Substation automated operation management system should include digital power production operation data and state records statistics, hierarchical data information, automated shunt exchange etc. The realization of these functions can through wireless sensing monitoring technology, intelligent information processing and judging technology.

(3) Automatic diagnosis technology. Monitoring device status information real-timely, by analyzing and comparing the 
equipment history preventive experimental data, operation data and online data, make smart decisions, given a report of the state of the equipment. Comprehensive management based on historical data, operation data and online monitoring data comprehensive management, according to the result of diagnosis formulate and adjust the maintenance plan.

\section{Distribution}

In power system, distribution connected with users directly and distributes electricity to users. As the concentrated reflection of all kinds of smart grid construction results, intelligent power distribution connect power users and smart grid.

From the current situation of distribution network in China, there are some problems as follows:

(1) The weak power grid structure restricting power supply ability. Distribution network automation application range and practical level is still low, monitoring and control ability of the power distribution system is limited.

(2) Since the enormous access of distributed energy and the future development trend, operation control and energy management of existing distribution network cannot meet the conditions of the intelligent. Reliability, security, quality of power supply and should be improved.

For the development of the intelligent power distribution, there are the following requirements:

(1) Distribution operation information monitoring. Take primary rack and equipment as the basis, power distribution automation system as the core, comprehensive use of various communication mode, to realize the monitoring and control of the power distribution system. Through integrating the related application system information, realize the scientific management of distribution system.

(2) Micro grid monitoring. Distributed energy, especially renewable energy such as solar energy, wind energy will access to grid, which will change the traditional energy structure. In order to achieve the safe and reliable power supply, implementation of supply-demand interaction, fully perception of the running status, the real-time measurement and online analysis, intelligent control, variable distribution equipment coordinate operation etc. should take into account.

(3) Reliable information acquisition ability. Combine various communication technology, strengthen assistance and fusion between sensor nodes, select high robust fusion algorithm, and improve the ability of acquisition.

(4) More quickly malfunction response, more reliable power supply, better power quality, have self-healing ability, reduce the malfunction loss and not affect the normal power supply situation.

\section{SMART GIRD AND LTE CHARACTERISTICS}

\section{A. Traditional LTE Traffic Characteristics}

A traditional LTE cellular network have following characteristics: (a) Sufficient bandwidth, all the primary countries in the world has allocated to LTE with a sufficient spectrum resources.

(b) Downlink dominated. Even though in LTE has some symmetrical uplink and downlink resource usage traffic such as VoIP conversations. However, in LTE network total downlink traffic exceeds total uplink traffic due to applications such as web browsing and video streaming (even when uplink intensive applications such as photo/video upload are taken into account). So the downlink traffic is the main data flow, and more subframes are allocated for downlink transmission in the TDLTE subframe configurations, e.g., the maximum downlink-touplink subframe ratio can reach 9:1, but the maximum uplinkto-downlink subframe ratio is only 3:2 [5].

(c) Mobility. In LTE, cell handover is a significant problem to provide a high QoS since UEs always moves such as when UEs on the high railway.

\section{B. Smart Grid Characteristics}

In smart grid, the communication system plays an important role in the management and control of the whole electrical grid. Compared to the traditional LTE cellular networks The Smart Grid has very different traffic characteristics.

(a) Uplink dominated. VoIP is unlikely to be a prominent application in the Smart Grid, and there are few other applications which are naturally symmetric in their uplink and downlink resource usage. The control center collects the sensing and measure data from power devices in a short interval so as to real-time manage and control the whole electrical grid. The uplink/downlink split of resource usage in a Smart Grid communications network is likely to be time variant to a larger extent than a traditional LTE network. For example, in peak periods of electricity demand, the Smart Grid operator may need to shed loads for network stability purposes or adapt the grid topology via switching to introduce a backup power generation facility, which clearly involves increasing the relative amount of downlink traffic.

(b) In wireless communications, the frequency spectrum is the most critical factor to support safe, efficient and reliable data transmission. However, most of "golden" spectrum resources have been allocated to a series of current wireless applications, such as radar, satellite, broadcast, GSM/3G/LTE, etc. Meanwhile, the amount of sensing and measure data per transmission is relatively small ( $<100$ bytes) in smart grid [8]. So it is almost impossible for the utility company to obtain so many spectrum resources dedicated for the electrical usage. If the smart grid networks share the unlicensed spectrum with other systems, the external interference will greatly impact on the reliability of data transmission.

(c) In smart grid, power devices are usually installed in a fixed location, such as smart meters, transformers, substations [9]. Hence, it is unnecessary to report the channel quality information to the control center frequently and refresh the scheduling process every $10 \mathrm{~ms}$ as in a traditional LTE system. Also, it can simplify or optimize mobility management, uplink timing synchronization and handover mechanisms in order to reduce power consumption and signaling overhead. Moreover, 
so the communication network can be designed into a fixed wireless access mode of the network, at the same time in the network the terminal roaming and less complex functions such as switch can be unconsidered, simplify the design of network structure and protocol. In smart grid communication network, generally speaking, power demand side communication terminal distributed uniformly., Power consumption size has nothing to do with the number of communication terminal, therefore, relatively simple solution can be used to realize the wireless resource allocation.

(d) The number of active power devices in a cell of smart grid is much larger than that in a general LTE cell, as stated in [10]. Notice that the traditional LTE cellular networks have been designed with human users in mind. Considering that most of mobile phones are usually in idle status, it only needs to support for tens or hundreds of channels per cell. In smart grid, however, all of thousands of power devices have their data to transmit, which require in the order of 10,000 channels per cell depending on the cell size and geographical area. As mentioned earlier, the smart grid network has limited bandwidth, so the number of power devices that can transmit simultaneously is constrained to a small amount. Due to a large number of power devices, it will bring unacceptable traffic delays for urgent services in smart grid, especially for TDD systems. Moreover, terminal are far apart from each other, therefore there are a higher requirements on wireless network coverage.

\section{LTE Characteristics}

The 3GPP Release 8 LTE standard supports both FDD and TDD duplexing modes [6]. There is purposefully a great deal of commonality between the two modes e.g. both employ OFDMA in the downlink and SC-FDMA in the uplink. This facilitates design re-use between FDD and TDD equipment and also makes dual mode FDD/TDD equipment economically viable. Most of the functional differences between the two modes relate to the physical layer and are detailed in [8][11][12].

Latency is a key issue for Smart Grid monitoring and control as the stability of the Smart Grid depends upon being able to detect and act on anomalies as quickly as possible. As illustrated in Fig. 2, the LTE uplink and downlink both utilize a $10 \mathrm{~ms}$ radio frame which is divided into ten $1 \mathrm{~ms}$ sub-frames. The sub-frame is the minimum resource allocation unit in the time domain, so the minimum latency possible is $1 \mathrm{~ms}$.

LTE TDD mode involves using a single unpaired frequency band for uplink and downlink, and switching in the time domain between uplink and downlink resources. A total of 8 TDD bands are defined in [5]. As uplink and downlink transmissions are not simultaneous, a TDD device does not need to support a duplex filter to separate the simultaneous uplink and downlink transmissions, thereby reducing the equipment cost.

One of the important advantages of TDD mode is that the relative allocation of uplink and downlink resources in the time domain can be skewed to match any arbitrary required traffic pattern. LTE TDD includes seven different uplink/downlink allocation configurations [5]. Note that an LTE frame of 10ms duration comprises ten sub-frames each of $1 \mathrm{~ms}$ duration.

LTE also supports the following channel bandwidths: 1.4 $\mathrm{MHz}, 3 \mathrm{MHz}, 5 \mathrm{MHz}, 10 \mathrm{MHz}, 15 \mathrm{MHz}$ and $20 \mathrm{MHz}$ to cater for spectrum allocations of different sizes in different bands and/or in different parts of the world [13]. It is expected that most Smart Grids will use channel bandwidths less than or equal to $10 \mathrm{MHz}$ and therefore TDD model is more suitable for smart grid communication.

\section{CONCLUSION}

The wireless communication network based on LTE or 4G is an appropriate solution for smart grid, which can effectively resolve the last mile access problem. In smart grid, the reliable data transmission is an essential to realize the automated management and control of all components connected to the grid. As a popular wireless broadband technology, the TD-LTE provides a competitive solution for relatively small bandwidth and asymmetric traffic in the smart grid networks. The cost of TD-LTE chipsets, terminals and system integrations will also be greatly reduced, when the TD-LTE networks are large-scale deployed around the world. The TD-LTE wireless communications system is an effective solution for wireless communications in smart grid.

\section{REFERENCES}

[1] SmartGrids SRA 2035 - Strategic Research Agenda,” European Technology Platform SmartGrids, European Commission, Tech. Rep.,Mar.2012.[Online]. http://www.smartgrids.eu/documents/20120308 sra2012.pdf

[2] P. Siano, C. Cecati, C. Citro, and P. Siano, "Smart operation of wind turbines and diesel generators according to economic criteria,” IEEE Trans. Ind. Electron., vol. 58, no. 10, pp. 4514-4525, Oct. 2011

[3] Z. Fadlullah, M. Fouda, N. Kato, A. Takeuchi, N. Iwasaki, and Y. Nozaki, "Toward Intelligent Machine-to-Machine Communications in Smart Grid,” IEEE Communication Magzine, vol. 49, No.4, pp:60-65.

[4] F. Li, W. Qiao, H. Sun, H. Wan, J. Wang , Y. Xia, Z. Xu and P. Zhang, "Smart Transmission Grid: Vision and Framework," IEEE Transaction on Smart Grid, vol. 1, No.2, pp:168-177

[5] 3GPP TR 25.913 V8.0.0 (2008-12), "Requirements for Evolved UTRA (EUTRA) and Evolved UTRAN (E-UTRAN)", Release 8

[6] Patel A., Aparicio J., Tas N., Loiacono M., Rosca J., "AssessingCommunications Technology Options for Smart Grid Applications",

[7] Smart Grid Communications (SmartGridComm), 2011 IEEE InternationalConference on, 2011, pp. 126-131

[8] R1-112912, “Overview on low-cost MTC UEs based on LTE,” Huawei, HiSilicon, CMCC, 3GPP RAN1\#66bis, Zhuhai, China, October 2011.

[9] R2-102340, "Smart grid traffic behaviour discussion,” Verizon, 3GPP RAN2\#69b, April 2010.

[10] A. Clark, C. J. Pavlovski, "Wireless networks for the smart energy grid: application aware networks,” Proceedings of IMECS 2010, Hong Kong, pp. 1243-1248, March 2010.

[11] 3GPP TS 36.212 V8.8.0 (2009-12), "Evolved Universal Terrestrial Radio Access (E-UTRA); Multiplexing and channel coding”, Release 8

[12] 3GPP TS 36.213 V8.8.0 (2009-09), "Evolved Universal Terrestrial RadioAccess (E-UTRA); Physical layer procedures”, Release 8

[13] 3GPP TS 36.101 V8.16.0 (2011-12), Evolved Universal Terrestrial Radio Access (E-UTRA); User Equipment (UE) radio transmission and reception, Release 8 